\title{
Index of names
}

Note: 'n.' after a page number refers to a note on that page.

Adorno, Theodor, 129, 147 n.23

Agamben, Georgio, 16, 166

Agnew, Jean-Christophe, 126

Althusser, Louis, 61

Arnold, Matthew, 3, 5, 48 n.9, 157

Aristotle, 44

Aughterson, Kate, 117 n.28

Bacon, Francis, 10, 89-90, 91, 101-14

Bahti, Timothy, 1, 7-8

Bass, Alan, 1

Bataille, Georges, 133, 150-1, 153

Baudrillard, Jean, 133-4, 148, 150-1, 153-4, 157-60, 162-5, 168 n.17, $168-9$ n. 20

Beardsworth, Richard, 155, 162, 165

Bennington, Geoffrey, 101, 116 n.24, 168 n. 12

Bentham, Jeremy, 48 n.9

Bhabha, Homi K., 155

Blanchot, Maurice, 150

Bogard, Richard, 168 n.17, 168-9 n.20

Bourdieu, Pierre, 10, 96-7, 100, 110-11, 124,129
Burt, Richard, 97-8, 100, 117 n.29

Caygill, Howard, 17 n.3

Clare, Janet, 100

Cohen, Walter, 56, 64, 99, 131

Critchley, Simon, 174-5

Daniel, Samuel, 54

de Man, Paul, 94-5, 107, 150, 172-5

Dekker, Thomas, 53

Deleuze, Gilles, 150

Derrida, Jacques, Aporias, 151, 154, 160-3

'The art of memoires', 172-6

'As if I were dead', 16, 148, 154-5, 164,166

'Freud and the scene of writing', 167-8 n. 12

The Gift of Death, 154, 158-60

Given Time, 11, 121-2, 134-42, 152

Glas, 174-5

'Living on/border lines', 155

'Mochlos', 1-2, 6-8, 12-13, 17, 21, 35,44 
The principle of reason', 2, 12, 43-

$$
6,49,79-80
$$

'Restitutions', 12, 22-3, 35-43, 46

$$
\text { n. } 2,47 \text { n. } 8,49,79
$$

'Sendoffs', 2

Specters of Marx, 150-1, 155-6, 161,163

'Violence and metaphysics', 122, $134,143-5,161$

Dollimore, Jonathan, 10, 52, 61-2, 64

Drayton, Michael, 66

Dutton, Richard, 100

Elam, Diane, 4

Evans, Walker, 23

Faulkner, Robert K., 104, 107, 110-11,113, 116n.23

Fichte, Johann Gottlieb, 5

Foucault, Michel, 52, 150

Freud, Sigmund, 47 n.8, 105, 151, 163

Genet, Jean, 174

Goldberg, Jonathan, 51-2

Goldstein, Kurt, 35-6

Gramsci, Antonio, 61

Greenblatt, Stephen, 52, 55-60, 130-2

Guattari, Felix, 150

Habermas, Jurgen, 77

Hall, Gary, 146 n.12

Hebdige, Dick, 127

Hegel, Wilhelm Friedrich, 5, 81-5, 150, 172-3, 175

Heidegger, Martin, 5, 21-43, 46 n.2, 47 n. $8,49,150,158,160-2,165-6,172$

Heinemann, Margot, 63, 99

Hobbes, Thomas, 54, 60

Hofstadter, Albert, 26, 38
Hoggart, Richard, 158

Holbein, Hans The Younger, 52

Holderness, Graham, 55

Holquist, Michael, 91-7, 103, 105, 145 6 n.9

Horkheimer, Max, 129

Howard, Jean, 57, 61

Humboldt, Wilhelm von, 2, 5

James I, 52-4, 66, 109

Jameson, Frederic, 21-5, 33, 40, 42, 58, 130, 132

Jonson, Ben, 53-4, 65, 97, 100

Kamuf, Peggy, 1, 4-5, 14, 15, 80-8

Kant, Immanuel, 1, 2, 5, 6-7, 13, 17 n.3, $21,35,85-7,94,96,146$ n.9, 154

Karpel, Craig, 88

Kierkegaard, Soren, 159

Kuhns, Richard, 26, 38

Lanson, Gustav, 4, 80, 81, 84-5

Lawson, Nigel, 63, 99

Leavis, F. R., 3, 61, 157

Levinas, Emmanuel, 143-4, 161, 166

Liu, Alan, 57

Llewelyn, John, 1

Locke, John, 81-2

Lyotard, Jean-Francois, 58, 76, 78, 101, 107,116 n.24, 130, 132, 136

Magritte, Rene, 23

Mallarme, Stéphane, 154

Marx, Karl, 150, 155

Mauss, Marcel, 121-2, 133-7, 141, 152-

$$
\text { 4, } 156
$$

Melville, Herman, 4, 80

Middleton, Thomas, 53-4, 66-7

Miller, J. Hillis, 4

Montrose, Louis, 57-8 
Mullaney, Steven, 100

Munch, Edvard, 23

Nava, Mica, 127, 129-30

Newbolt, Henry, 3, 5

Newman, John Henry Cardinal, 3,5

Nietzsche, Friedrich, 5, 150

Orgel, Stephen, 52

Patterson, Annabel, 100

Peguy, Charles, 4, 80

Plato, 154

Pye, Christopher, 60, 109, 125-6

Rand, Richard, 1

Rawley, William, 103, 113

Readings, Bill, 2-4, 14, 73-80, 81, 89, 174

Royle, Nicholas, 167 n.2

Schelling, Friedrich Wilhelm Joseph von, 5

Schiller, Friedrich von, 2, 5

Schleiermacher, Friedrich, 5

Shakespeare, William, 55-6, 59, 63, 99, 131
Shapiro, Meyer, 21-3, 35-41, 43, 46

$$
\text { n. } 2,47 \text { n. } 8,49
$$

Sharpe, Kevin, 100

Siegel, James, 2

Sinfield, Alan, 10

Stanton, Domna C, 93-4, 96, 127-9, 146 n.9

Taine, Hippolyte, 4, 81, 84

Thompson Klein, Julie, 123-4

Van Gogh, Vincent, 13, 15, 17, $21-3,26,28-30,33-43,46$ n. $2,49,171$

Velasquez, Diego Rodriguez de Silva y, 52

Warhol, Andy, 23-4

Wayne, Don E., 58

Weinberger, Jerry, 104, 106-7

Wormald, B. H. G., 103

Wortham, Simon, 117 n.27

Wynne-Davies, Marion, 55

Young, Robert, 1, 9, 42, 47 n.9, 61-3 